\title{
Utilização da tecnologia NIRS para predição dos valores nutricionais de forrageiras
}

\author{
Ângela Fonseca Rech ${ }^{1}$ e Simone Silmara Werner ${ }^{2}$
}

\begin{abstract}
Resumo - A Espectroscopia no Infravermelho Próximo (Near Infrared Spectroscopy - NIRS) tem sido muito utilizada com objetivo analítico nas mais diversas áreas de pesquisa, produção e indústria em materiais como petróleo, produtos químicos, óleos, madeiras, polímeros, tecidos, medicamentos, alimentos, bebidas, solos etc. Após a correta implantação da técnica, a análise não necessita de reagente químico, é robusta, rápida, não destrutiva e não exige mão de obra especializada. Porém, para que a técnica seja utilizada de forma confiável, modelos de calibração precisos e acurados devem ser desenvolvidos para cada material e para cada componente de interesse. O objetivo deste trabalho é fornecer algumas informações básicas importantes sobre a técnica NIRS, seu fundamento, potencial e limitações de uso.
\end{abstract}

Termos para indexação: Modelos Calibração; Bromatologia; Espectroscopia.

\section{Use of NIRS technology for prediction of nutritional values of forages}

\begin{abstract}
Near Infrared (NIR) Spectroscopy (NIRS) has been widely used for analytical purposes in the most diverse areas of research, production and industry in materials such as petroleum, chemicals, oils, wood, polymers, textiles, food, beverages, soils, etc. After the correct implantation of the technique, the analysis does not require chemical reagent, it is robust, fast, it is not destructive and does not require specialized labor. However, for the technique to be used reliably, precise and accurate calibration models must be developed for each material and each component of interest. Some important information about NIRS, fundamentals, potential and limitations of use are presented in this work.
\end{abstract}

Index terms: Calibration Models; Bromatology; Spectroscopy.

\section{Introdução}

Os avanços nas áreas computacional e eletrônica ocorridos na década de 1980 permitiram que as informações obtidas com o espectro coletado na região do infravermelho próximo fossem tratadas e analisadas corretamente, popularizando a Espectroscopia no Infravermelho Próximo (Near Infrared Spectroscopy - NIRS) como técnica analítica. Esta técnica tem sido utilizada para determinação da composição química de um grande número de materiais e é particularmente indicada para aqueles que contenham as ligações químicas $\mathrm{C}-\mathrm{H}$, O-H, N-H, S-H e C=O (PASQUINI, 2018), exemplificadas na Tabela 1.

Norris et al. (1976) foram os primeiros a relatar que a espectroscopia NIR, associada a métodos quimiométricos, poderia prever de forma rápida e razo-

Tabela 1. Regiões espectrais dos respectivos grupos químicos comumente vistos em NIRS Table 1. Spectral regions of the respective chemical groups commonly seen in NIRS

\begin{tabular}{ccc}
\hline Grupo & $(\lambda), \mathbf{n m}$ & $(\bar{v}), \mathbf{c m}-\mathbf{1}$ \\
\hline $\mathrm{CH}_{3}$ & $2275-2285$ & $4400-4380$ \\
$\mathrm{NH}_{2}$ & $1520-1540$ & $6580-6490$ \\
$\mathrm{H}_{2} \mathbf{O}$ & $1930-1940$ & $5280-5150$ \\
$\mathrm{C}=\mathbf{O}$ & 1950 & 5130 \\
$\mathbf{C}=\mathrm{CH}_{2}$ & $860-870$ & $11630-11490$ \\
\hline
\end{tabular}

$\bar{\lambda}$-Comprimento de onda; $\bar{v}$-Números de onda

Fonte: Adaptado Siesler et al., 2001

$\lambda$-Wavelength; $\bar{v}$-Wavenumbers

Source: Adapted Siesler et al., 2001

ável a qualidade das forragens. Porém, sua precisão e acurácia dependem de bons modelos de calibração. Yang et al. (2017) realizaram um trabalho para desenvolvimento e calibração dos modelos para a previsão de parâmetros de qualidade de forragem em Lolium multiflorum. Os autores relataram que a técnica NIRS foi eficiente para predizer os valores de proteína bruta (PB), fibra em detergente ácido (FDA), fibra em detergente neutro (FDN) e carboidratos solúveis em água. Além disso, a técnica proporcionou uma economia de tempo e custos associados à análise que, segundo eles, foi de apenas $20 \%$ quando comparada à metodologia tradicional.

A construção dos modelos de calibração para NIRS necessita que um número considerável de amostras esteja

Recebido em 27/9/2018. Aceito para publicação em 5/4/2019.

${ }^{1}$ Zootecnista, MSc., Pesquisadora, , Epagri, Estação Experimental de Lages, Rua João José Godinho, S/N, Bairro Morro do Posto, Lages - SC, CEP 88502-970

Lages, SC, CP 181, fone: (49) 3289-6414, e-mail: angelarech@epagri.sc.gov.br.

${ }^{2}$ Matemática, Dr., Pesquisadora, Epagri, Estação Experimental de Lages, e-mail: simonewerner@epagri.sc.gov.br 
disponível, seja analisado pelos métodos de referência e que os resultados obtidos estejam distribuídos em uma escala abrangente. Essas amostras são submetidas a leituras espectrais (Figura 1) e os dados das leituras são cruzados com os valores obtidos pelos métodos de referência. Para isso, no entanto, é necessário definir o método de regressão que será empregado, o melhor prétratamento matemático nos dados espectrais, o segmento do espectro a ser utilizado, entre outros.

\section{Fundamento}

A NIRS é um tipo de espectroscopia vibracional que utiliza a energia de fótons na faixa do comprimento de onda de 750 nanômetros $(\mathrm{nm}$ ) a $2500 \mathrm{~nm}$ (ou número de onda entre 13333 a $4000 \mathrm{~cm}^{-1}$ ) e sua interação com a matéria. A onda eletromagnética produzida pelo espectrofotômetro de infravermelho próximo NIR (Near Infrared) (Figura 2) ao incidir na amostra a ser analisada, é parcialmente absorvida e refletida. As ligações covalentes das substâncias orgânicas absorvem essa energia que é transformada em vibração das ligações moleculares. A radiação que não é absorvida é refletida, analisada e quantificada. Entre os modos de medição, predominam aqueles baseados na refletância difusa para materiais sólidos (moídos, granulados ou intactos) (PASQUINI, 2018).

A refletância difusa é resultado da incidência da radiação na superfície do material, com subsequente penetração na camada superficial das partículas excitando modos vibracionais das moléculas da amostra seguidos pelo espalhamento da radiação em todas as direções (Figura 3). Os espectros obtidos (intensidade de refletância em função do comprimento de onda) (Figura 1) contêm informação indireta da absorção, espaIhamento e reflexão da luz original irradiada. A absorção e o espalhamento da radiação são os principais fenômenos que afetam o espectro de refletância (PASQUINI, 2018).

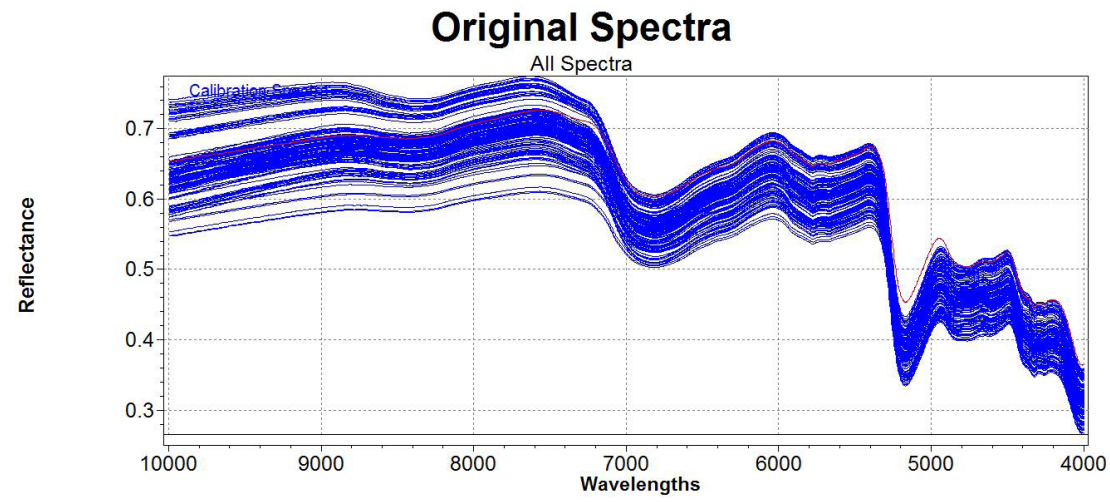

Figura 1. Espectros na região do infravermelho proximal de amostras de azevém Figure 1. Spectrums in the proximal infrared region of ryegrass samples

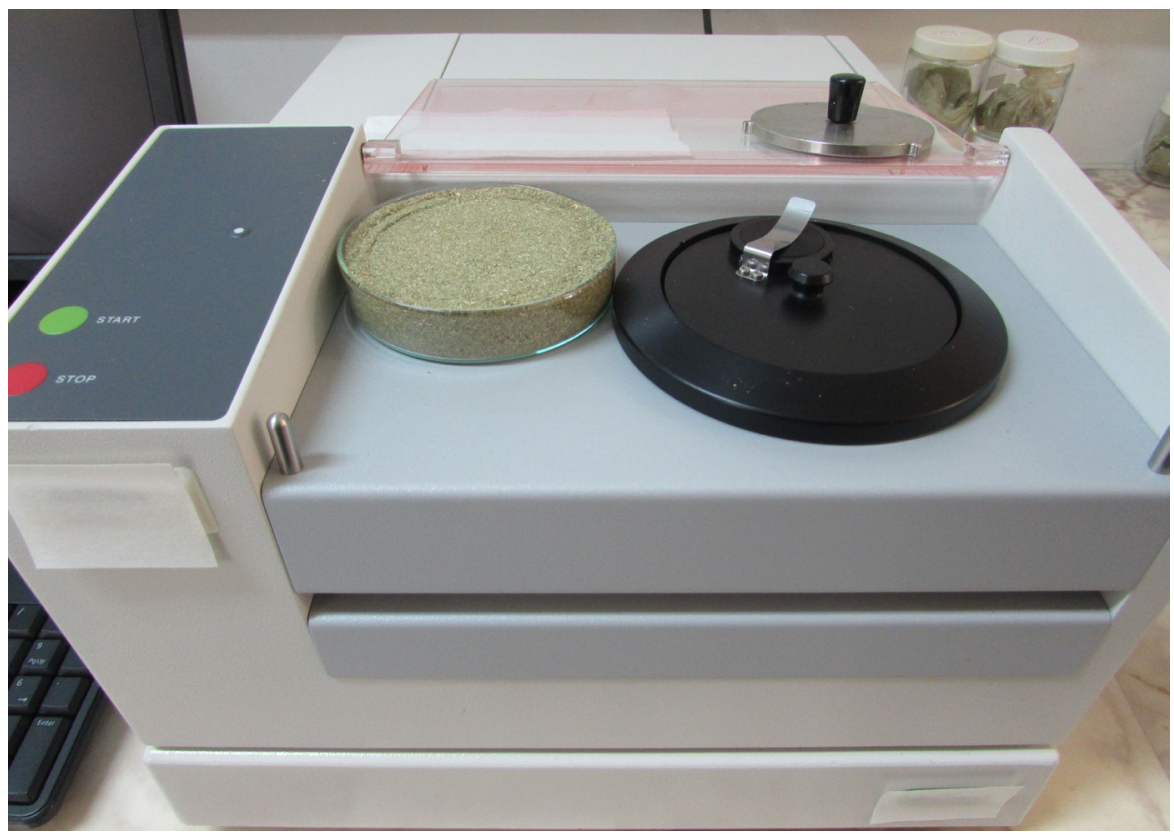

Figura 2. Espectrofotômetro de infravermelho próximo NIR. Foto: Angela F. Rech Figure 2. NIR Near Infrared Spectrophotometer. Photo: Angela F. Rech

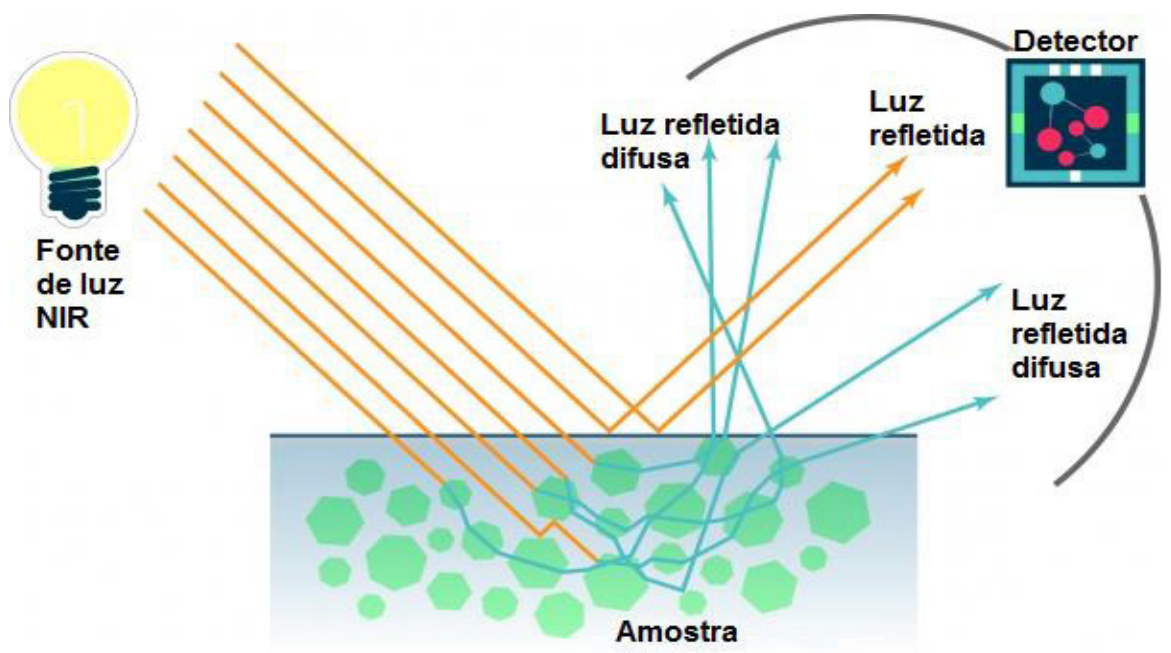

Figura 3. Refletância difusa

Fonte: Adaptado de Macdonald, 2016;

Figure 3. Diffuse Reflectance

Source: Adapted Macdonald, 2016 
Os componentes orgânicos dos alimentos possuem características de absorção específicas, onde ocorrem vibrações das ligações hidrogenadas induzidas pelo calor nos grupos funcionais das moléculas. Esta absorção de energia é utilizada para estimar o número e o tipo de ligações moleculares das amostras. Assim, pela espectroscopia NIR há condições de predizer o conteúdo dos diferentes componentes nutricionais, por meio de equações de calibração para cada um desses componentes e para diferentes alimentos (MARTEN et al., 1985). Porém, devido à complexidade e à quantidade de informações contidas nos espectros gerados é muito difícil fornecer interpretações quantitativas diretas, sendo fundamental a construção cuidadosa de modelos de calibração e validação antes de utilizar a espectroscopia NIR como técnica analítica (MONTEIRO et al., 2017).

A calibração é a construção de um modelo estatístico que irá estimar o teor de um componente no material de interesse, a partir da absorção da energia pela amostra submetida à radiação na faixa do infravermelho proximal. Para otimizar a utilização das informações contidas nos espectros no infravermeIho proximal, técnicas multivariadas são usadas para remover fontes de variação indesejáveis causadas por diferenças no tamanho, na forma das partículas e na densidade das amostras, espalhamento da luz, ruídos e efeitos aditivos na linha de base. Os pré-tratamentos matemáticos são importantes também para reduzir o número de fatores necessários, simplificar o modelo de calibração, dar maior ênfase aos picos e melhorar a interpretação do modelo. É necessário ainda detectar e excluir as amostras aberrantes (outliers) discrepantes que não se encaixam ou não correspondem à calibração (MARTEN et al., 1985).

O método de regressão mais utilizado para desenvolvimento de modelo de calibração e predição dos teores em ma- teriais agropecuários (milho, soja, café, carnes, plantas, forragens, madeira, carvão etc.) é o Partial Least Squares (PLS). Os pré-tratamentos matemáticos muito utilizados são as derivadas de $1 \underline{a}$ e $2 \underline{a}$ ordem, Multiplicative Scatter Correction (MSC), Standard Normal Variate (SNV) e filtro de Savitzki-Golay, assim como as combinações dos mesmos (PASQUINI, 2018).

\section{Etapas necessárias}

Para obter sucesso na utilização desta tecnologia é necessário seguir algumas etapas: selecionar amostras representativas e que possuam grande variabilidade em relação à concentração do componente que se deseja determinar; obter bons resultados analíticos pelos métodos de referência convencionais; realizar as leituras espectrais; aplicar os tratamentos matemáticos; desenvolver modelos estatísticos; validar com amostras não utilizadas na construção do modelo; avaliar os resultados; e implantar a rotina analítica no laboratório.

\section{Utilização}

A técnica NIRS tem sido adotada como método oficial, método 930.03, pela Association of Official Analytical Chemists, na predição do teor de proteína bruta e FDA em forragens (Association of Official Analytical Chemists, 1984). Muitos trabalhos e artigos utilizando espectroscopia NIR têm sido publicados e, segundo Williams et al. (2017), a maioria desses estudos não informa alguns itens importantes que precisam ser relatados necessários para quem procura duplicar a aplicação ou para revisores do trabalho. Estes mesmos autores sugerem que indicadores como o erro padrão da validação cruzada Standard Error of Cross-Validation (SECV) ou erro médio quadrático da validação cruzada Root Mean Square Errors of Cross-Validation (RMSECV), erro mé- dio quadrático de predição Root Mean Square Error of Prediction (RMSEP), coeficiente de determinação $\left(R^{2}\right)$ da validação ( $R^{2}$ val), desvio, entre outros, sejam relatados no artigo.

O desempenho dos modelos pode ser avaliado pelo RMSEP e R2 dos valores previstos e de referência, e pela Relação do Desvio de Previsão Ratio of Prediction Deviation (RDP), como recomendado por Williams \& Sobering (1993). O RDP é a relação entre o erro padrão de previsão com o desvio padrão dos dados originais e é utilizado para a avaliação e monitoramento de calibrações.

Utilizando a espectroscopia NIR, Fontaneli et al. (2002) desenvolveram modelos de calibração para predição de propriedades nutricionais de silagem de milho e concluíram, com base no $\mathrm{R}^{2}$, erro padrão da validação cruzada, erro padrão da validação e $R^{2}$ val que os modelos desenvolvidos apresentavam elevada acurácia.

Segundo Fernandes (2015), a técnica NIRS, associada ao método de calibração multivariada de regressão por meio de quadrados mínimos parciais, mostrou-se eficiente para prever a composição químico-bromatológica de vagem de algaroba e da palma forrageira. Este mesmo autor escolheu os melhores modelos com base nos resultados de RMSEC, RMSECV e R².

Com objetivo de ajustar um modelo de calibração multivariada para a predição do teor de proteína bruta em biomassa de capins braquiária, Neto et al. (2012) desenvolveram e avaliaram os modelos pelos resultados obtidos para o Root Mean Square Error of Calibration (RMSEC) Erro Médio Quadrático de Calibração, RMSECV, RMSEP e $\mathrm{R}^{2}$. Concluíram que o modelo de calibração multivariada para a análise do teor de proteína em biomassa de braquiária foi adequado e poderá ser adotado como método de rotina de análise. Neto et al. (2009) concluíram que a espectros- 
copia NIR é uma técnica alternativa viável para a determinação do teor de proteína total e matéria seca em amostras de milho moído. Os modelos ajustados para proteína bruta, matéria seca e fósforo apresentaram performances adequadas para utilização em amostras provenientes de ensaios de screening (para triagem de amostras) ou onde se tem grande número de repetições de amostras por tratamentos. Para utilização em determinações analíticas como método de rotina laboratorial, no entanto, os modelos de calibração precisavam ser aprimorados.

A técnica também tem sido utilizada como ferramenta para desenvolver modelos de predição de consumo de matéria seca e orgânica, matéria orgânica digestível, digestibilidade aparente, qualidade de ingredientes em indústrias, entre outras.

\section{Limitações}

Apesar das vantagens apresentadas neste informativo, a espectroscopia NIR apresenta algumas limitações: Métodos de referência analítica reconhecidos são necessários para fornecer os resultados exigidos na etapa de modelagem; O modelo geralmente é de difícil interpretação química sendo necessária a utilização de algoritmos; Os modelos desenvolvidos precisam ser atualizados com frequência; Uma modelagem robusta pode exigir centenas ou até milhares de amostras pré-analisadas pelo método de referência; A técnica não é muito sensível e o limite analítico geral de quantificação, usualmente considerado, está na faixa dos 5000ppm ou 0,5\%, sob condições favoráveis (PASQUINI, 2018). Isso pode restringir sua aplicação às substâncias encontradas em concentrações superiores a 0,5\%.

\section{Considerações finais}

A espectroscopia NIR apresenta grandes vantagens em relação aos mé- todos de análises tradicionais para predição dos valores nutricionais de forrageiras, mas tem como fatores limitantes a necessidade de um grande número de amostras já analisadas pelos métodos de referências e a necessidade de ajuste de bons modelos de calibração. Cumpridas essas exigências o método poderá ser utilizado na rotina laboratorial para aumentar a capacidade de análises e reduzir o volume de reagentes e de resíduos gerados nas análises químicas convencionais.

\section{Referências}

ASSOCIATION OF OFFICIAL ANALYTICAL CHEMISTS. Official methods of analysis. 14 ed. Washington, DC: AOAC, 1984. 1019p.

FERNANDES, A. M. F. Uso da espectroscopia de reflectância do infravermelho próximo (nirs) para previsão da composição bromatológica de vagens de algaroba e palma forrageira. 2015. 105f. Dissertação (Mestrado em Zootecnia) - Universidade Estadual Vale do Acaraú, Sobral, CE, 2015.

MACDONALD, C. Canadian mills opt for FITNIR analyzers. Pulp \& Paper Canada. 2016. Disponível em: https://www.pulpandpapercanada.com/news/canadian-mills-opt-forfitnir-analyzers-1100000232/. Acesso em: 24 out. 2018.

FONTANELI, R.S.; DURR, J.W.; SCHEFFERBASSO, S.M.; HAUBERT, F.; BORTOLINI, F. Validação do método da reflectância no infravermelho proximal para análise de silagem de milho. Revista Brasileira de Zootecnia, v.31, n.2, p.594-598, 2002.

MARTEN, G. C.; SHENK, J. S.; BARTON II, F. E. Near infrared reflectance spectroscopy (NIRS), analysis quality. Washington, D.C.: USDA, $1985.110 \mathrm{p}$.

MONTEIRO, A. R. D, FEITAL, T. S., PINTO, J. C. Statistical Aspects of Near-Infrared Spectroscopy for the Characterization of Errors and Model Building. Applied Spectroscopy. v.71, n.7, p.1665-1676, 2017. Disponível em: https://www.ncbi.nlm.nih.gov/pubmed/28447492. Acesso em: 20/10/2018.

NETO, M. M. G.; SIMEONE, M. L. F.; GUIMARÃES, C. C.; VASCONCELOS, F. V.; UBA, M. A. Predição de proteína, matéria seca e fósforo em grãos de milho pela espectroscopia de reflectância no infravermelho próximo. Boletim de Pesquisa e Desenvolvimento n.9, Embrapa Milho e Sorgo, Sete Lagoas, MG, 2009. 19p.

NETO, M. M. G.; SIMEONE, M. L. F.; GUIMARÃES, C. C. Predição do teor de proteína bruta em biomassa de capins braquiária por meio de espectroscopia NIR. Comunicado técnico n.205, Embrapa Milho e Sorgo, Sete Lagoas, MG, 2012. 5 p.

NORRIS, K. H.; BARNES, R. F.; MOORE, J. E.; SHENK, J. S. Predicting forage quality by near infrared reflectance spectroscopy. Journal of Animal Science, Champaign, v.43, n.4, p.889-897, 1976.

PASQUINI, C. Near infrared spectroscopy: A mature analytical technique with new perspectives - A review. Analytica Chimica Acta, v.1026, n.5, 2018, p.8-36, 2018.

SIESLER, H. W.; OZAKI, Y.; KAWATA, S.; HEISE, H. M. Near-Infrared Spectroscopy. Principles, Instruments, Applications. 3 ed. Weinheim: Wiley - VCH, 2001. 361p.

WILLIAMS, P.C.; SOBERING, D.C. Comparison of commercial near infrared transmittance and reflectance instruments for analysis of whole grains and seeds. Journal of Near Infrared Spectroscopy, v.1, p.25-32, 1993.

WILLIAMS, P.; DARDENNE, P.; FLINN, P. TUtorial: Items to be included in a report on a near infrared spectroscopy project. Journal of Near Infrared Spectroscopy, v.25, n.2, p.85-90, 2017.

YANG, Z., NIE G., PAN L., ZHANG Y., HUANG L., MA X., ZHANG, X. Development and validation of near-infrared spectroscopy for the prediction of forage quality parameters in Lolium multiflorum. PeerJ, v.5, e3867, 2017. 